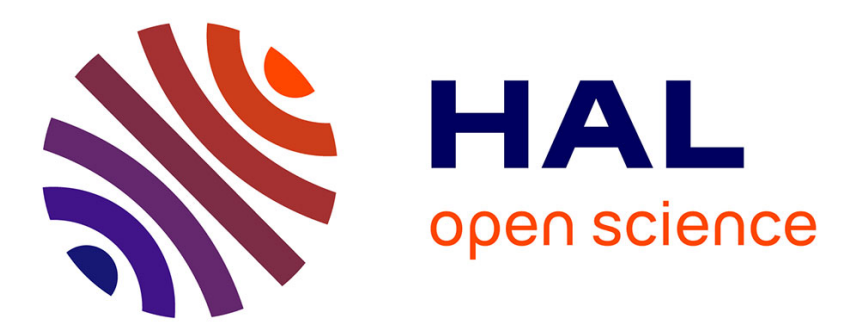

\title{
Low Latency of Re-Authentication During Handover : Re-Authentication Using a Signed Token in Heterogeneous Wireless Access Networks
}

Hassane Aissaoui, Pascal Urien, Guy Pujolle

\section{- To cite this version:}

Hassane Aissaoui, Pascal Urien, Guy Pujolle. Low Latency of Re-Authentication During Handover : Re-Authentication Using a Signed Token in Heterogeneous Wireless Access Networks. 10th International Conference on Wireless Information Networks and Systems - WINSYS 2013, Jul 2013, Reykjavik, Iceland. pp.1-7. hal-01018179

\section{HAL Id: hal-01018179 \\ https://hal.science/hal-01018179}

Submitted on 3 Jul 2014

HAL is a multi-disciplinary open access archive for the deposit and dissemination of scientific research documents, whether they are published or not. The documents may come from teaching and research institutions in France or abroad, or from public or private research centers.
L'archive ouverte pluridisciplinaire HAL, est destinée au dépôt et à la diffusion de documents scientifiques de niveau recherche, publiés ou non, émanant des établissements d'enseignement et de recherche français ou étrangers, des laboratoires publics ou privés. 


\title{
Low Latency of Re-Authentication During Handover: Re-Authentication Using a Signed Token in Heterogeneous Wireless Access Networks
}

\author{
Hassane AISSAOUI ${ }^{1}$, Pascal URIEN ${ }^{1}$ and Guy PUJOLLE ${ }^{2}$ \\ ${ }^{I}$ Network and Computer Science Department, TELECOM-ParisTech : LTCI CNRS Laboratory, rue Barrault, Paris, France \\ ${ }^{2}$ LIP6-University Pierre and Marie Curie Paris VI : CNRS Laboratory, 4 Place Jussieu, Paris, France \\ \{hassane.aissaoui, pascal.urien\}@telecom-paristech.fr, guy.pujolle@lip6.fr
}

Keywords: Wirless, Roaming, Handover, Digital Signature, Signed Token, Trusted Infrastructure, (Re)-authentication, (Re)-association, Mobility Anytime Anywhere, Ubiquitous Access, Low Latency, Real-Time Applications, AAA Framework, IEEE802.1x/EAP Protocol,

Abstract: Wireless networks provide several advantages over wired networks. They offer: a satisfactory bandwidth, mobility, easy deployment in difficult areas, long-term savings and the speed more and more higher. However, they also have some disadvantages in regard to security, performances during re-authentication, execution of real-time applications and interference from other electromagnetic sources (Bluetooth, microwave, etc.).

The existing solutions to reduce delays of Handover intercellular are specific solutions to a particular network or manufacturer of this technology.

The main objective of this paper is to propose novel mechanisms based on digital signatures to obtain low latency re-authentication during Handover in Wireless Access Networks. Our infrastructure will be based on trusted relationship between the heterogeneous access points and the authentication servers, in order to allow the mobility anytime anywhere to any user, and the ubiquitous access to the Future Internet, while ensuring the right level of security to both the end users as well to the wireless networks.

In this context, the main issues are to resolve the seamless Handover and the re-authentication during mobility of station. By using a signed token in our trusted infrastructure, we achieve a unique authentication and a fast re-authentication. Therefore, the requests to the authentication server are considerably limited.

\section{INTRODUCTION}

Unlike the Global System for Mobile Communications (GSM), the concepts of "Roaming and Handover" represent different actions.

In general, the process of handover is natively implemented in Mobile Station (MS), Base Station Controller (BSC) and Mobile Switching Center (MSC). The handover process in GSM involves four steps: the measurement, the request, the decision and the execution of handover. The request of the handover is generated by the BSC, and the decision is provided by the MSC. the other steps can be maintained by the BSC.

In wireless technologies, the Handover is a process that represents a mobility of the mobile node
(MN) to another target Point of Attachment (PoA), which offers a higher radio link quality.

The Handover is a fundamental mechanism in the inter-cellular communication; involving a set of operations that allows the mobile station to change the PoA without losing the connectivity and the session. These operations generate several steps and every single step causes delays and has an impact on the real-time applications such as Voice over IP (VoIP).

The rest of this paper is structured as follows: In (Section 2), we present an overview of the constraints and the causes of the inter-cellular delays and the impact of security on the Handover. In (Section 3), we examine proprietary solutions and improvements to the Wireless Local Area Networks (WLAN) or IEEE 802.11 Standards (WLAN 1997). 
We will analyze, (Section 4), the WLAN Standards and Authentication, Authorization and Accounting Framework (AAA, 2000) which is based on Extensible Authentication Protocol (EAP 2004). In (Section 5), we illustrate the advantages and properties of digital signature that allow privacy and authenticity of a message, freshness against replay attacks / anti-replay, non-repudiation and anonymity (ISO 7498-2:1989). In (Section 6), we present our contribution which involves the use of asymmetric cryptography to sign tokens after client authentication, how this token save the context of a user session and how the stations perform a fast reauthentication during a Handover with this token?

Finally, we present in (Section 7), a conclusion and perspectives for future work.

\section{INTERCELLULAR HANDOVER PROBLEMS}

\subsection{Delays Due to Technology IEEE 802.1X Protocol}

Delays due to Handover have been for a long time a recognized problem in wireless networks.

Certain experimental studies (Mishra et al., 2003) \& (Velayos and Karlsson, 2004), attributed the delay in the IEEE 802.11 networks to the following phases: scanning, re-initialisation, reassociation and re-authentication, during which a wireless station discovers neighboring access points.

According to (Zrelli and Shinoda, 2007), the use of EAP authentication causes significant delays regarding the re-authentication phase. Indeed, the EAP is a component of the AAA Framework to secure and control access to different networks.

In this centralized Framework, the delays due to the EAP protocol, during re-authentication phase of a mobile station can become a problem, especially in the use of real-time applications.

When the mobile station moves to a new AP, it loses its attributes and continuity of the session, and must re-authenticate again with the centralized Framework. This process of the re-authentication greatly increases latency during the Handover.

\subsection{Constraint of Real-Time Applications}

VoIP is a real-time application, which imposes itself as the main application to test the validity of the performance of Handover in the wireless network. Indeed, the main constraint of VoIP is the continuity of the flow so that there is no break in the conversation (da Conceicão et al., 2006), because human ear perceives a break of the voice greater than $50 \mathrm{~ms}$ during a VoIP communication.

\section{OVERVIEW OF SOLUTIONS THAT IMPROVE HANDOVER}

In real-time applications, it is important to reduce the time of re-authentication and re-association during the Handover process while supporting advanced security mechanisms.

Table 1: Effect of roaming on Packet loss (Exact copy from a Source: Bangolae et al. 2006, p.6)

\begin{tabular}{|c|c|c|c|}
\hline $\begin{array}{c}\text { Authentication } \\
\text { method }\end{array}$ & $\begin{array}{c}\text { Average } \\
\text { Roaming } \\
\text { time (ms) }\end{array}$ & $\begin{array}{c}\text { Average } \\
\text { Packet } \\
\text { Loss \% }\end{array}$ & $\begin{array}{c}\text { Maximum } \\
\text { Consecutive } \\
\text { lost datagrams } \\
\text { (Average) }\end{array}$ \\
\hline $\begin{array}{c}\text { Baseline - Full } \\
802.1 \mathrm{x} \text { EAP } \\
\text { Authentication }\end{array}$ & 525 & 1.8 & 53 \\
\hline $\begin{array}{c}\text { Fast Transition } \\
\text { using 802.11r }\end{array}$ & 42 & 0.2 & 6 \\
\hline
\end{tabular}

Many enhancements have been made to the 802.11 standard (IEEE 802.11i), but it is not easily adaptable to the mobility of real-time applications (Bangolae et al., 2006). Implementing a caching mechanism in 802.11i has not improved the time roaming, it is around 350 milliseconds. This time of switching to a new AP is 7 times higher than the maximum latency for VoIP (which is about $50 \mathrm{~ms}$ ).

In (Table 1), it shows the average time roaming (525 ms) and packet loss (1.8\%) using basic authentication $802.11 \mathrm{i}$, especially when there is a traffic load of the back-end (eg: Remote Authentication Dial In User Service) (RADIUS, 2000).

The first solution developed to reduce the latency in Handover is the Inter Access Point Protocol (IAPP). Based on IAPP, the Institute of Electrical and Electronics Engineers (IEEE) in 2003 led to the drafting of a "Trial-Use Recommended Practice" which presents the 802.11f (IAPP, 2003). The deployment of this recommendation has been virtually no communication. Despite this ratification, 
the manufacturers have continued to implement their methods of Roaming in their wireless networks technologies. It was abandoned in 2006 for various reasons, in particular the very slow Handover latency.

\section{IEEE 802.1X / EAP AND AAA FRAMEWORK}

\subsection{IEEE 802.1X}

The Port Based Access Control or IEEE 802.1X protocol is developed by the IEEE based on the control ports (IEEE 802.1X, 2001). It allows access to a network after authentication from a wired or wireless network. The main components of this mechanism are presented as following:

- Supplicant: the system that will be authenticated (station: STA).

- Authentication Server (AS): that manages user accounts (AAA Framework, RADIUS).

- Authenticator: network equipment that plays the role of mediator between the STA (Access Point, Switch ...) and AS.

Each AP shares a secret with AS, to enable them to perform mutual authentication and establish a secure channel.

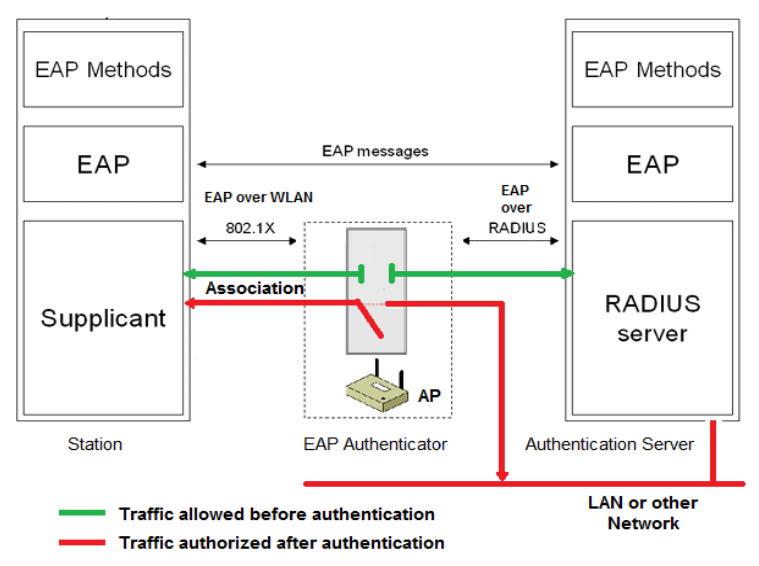

Figure 1: 802.1X architecture of authentication.

The STA also shares a secret with the AS, usually it is an username and password, as the STA is not authenticated, $802.1 \mathrm{X}$ does not allow it access to the network, only exchanges related to authentication processes are relayed to the AS by the AP. Once the STA is authenticated, the AP permits the traffic related to this STA "Figure 1".

\subsection{IEEE 802.1i and EAP Protocol}

IEEE $802.1 \mathrm{X}$ is based on EAP authentication methods that are specified in the RFC 3748 standard and respect the requirements of the standard RFC 4017 , in order to provide mutual authentication between a station (STA) and the local network. There are two kinds of traffic EAP "Figure 1" :

- EAP over LAN (EAPoL) or EAP over WLAN $(\mathrm{EAPoW})$ : EAP is transported in these specific frames between the Supplicant (STA) and Authenticator (AP).

- EAP over RADIUS (EAPoRADIUS) : are the frames transported between the AP and the authentication server (AS). The dialogue between both is a simple "re-encapsulation" of EAP packets without modifying the contents of the package by the AP.

EAP is a transport protocol for authentication. It defines mechanisms for exchanging authentication between devices. In order to get the access to a wireless network infrastructure, the STA must be authenticated.

As shown in phase 0, "Figure 2": the first step is, of course, the association 802.11, of the STA to AP, which must be done previously at $802.1 \mathrm{X}$ authentication phase.

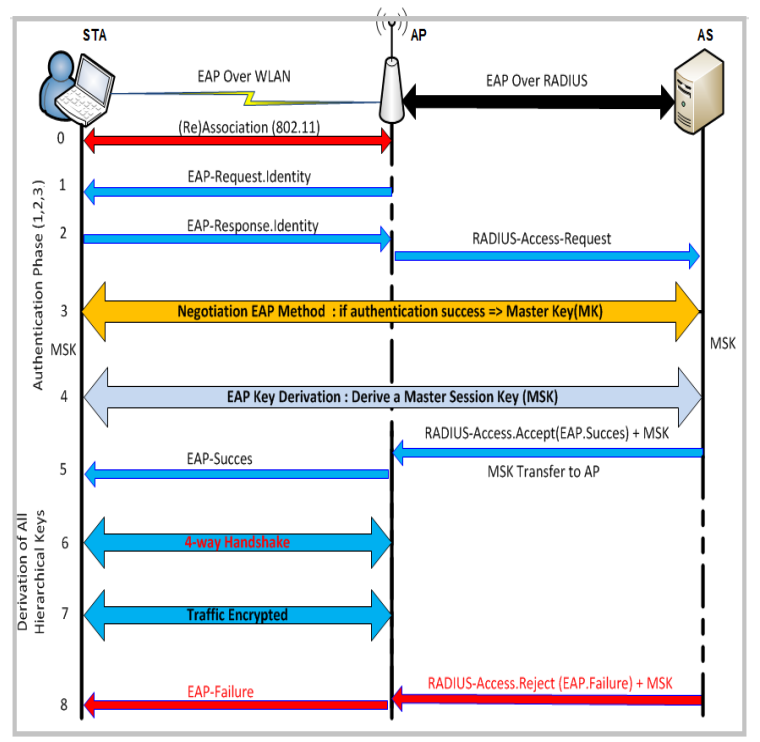

Figure 2: EAP Exchanges.

The authentication phase is then initiated via traffic (EAPoW):

- Phase 1: (EAP-Request.Identity) sending a request from the AP to the STA. 
- Phase 2: (EAP-Response.Identity) is STA response to the request, and attaching a first identifier (EAP-ID). This response is retransmitted to the RADIUS server via (EAP Over Radius) in the request (Access.Request).

- Phase 3: From this time, the second stage begins exchanges that depend on the chosen authentication method (EAP-TLS, EAP-TTLS, EAP-MD5, LEAP, etc.). Therefore EAP messages (requests and responses) are exchanged between the RADIUS server and the STA. If the STA is authenticated, the AS and the STA negotiate a Master Key (MK). This MK is only valid for this session between the STA and the AS. The access point plays only a passive relay at the moment.

- Phase 4: After successful authentication, the STA and the AS derive from the MK a secret key called "Master Session Key" (MSK), during one of the last messages sent by the AS. This key is known only by the client and the server. The MSK can be derived only by the client and the AS.

- Phase 5: At the end of these exchanges AS indicates to the AP the success of this procedure with EAP-Success message. Then, the AS transfers the MSK to the AP in the Access.Accept packet in the attribute "Microsoft Vendor-specific RADIUS Attributes" (RFC 2548, 1999) and (RFC 3078, 2001). This Attribute contains (MS-MPPERECV-KEY), a session key for use by the Microsoft Point-to-Point Encryption Protocol (MPPE). This key is intended for encrypting packets received by the Authenticator from the remote STA. It is included in RADIUS-AccessAccept packets. From this moment, the server has no interaction and the exchanges continue between AP and STA. The AP takes decisions on access control LAN instead of the server.

- Phase 6: The MSK is used as the symmetric key, which is valid only for this session between the STA and the AP. This symmetric key will be derived in three keys between the STA and the AP, using the protocol "4-way handshake". Especially, the Group Transcient Key (GTK) shared by all stations connected to the same AP. It is only used to secure the Multicast and Broadcast in the Basic Service Set (BSS). It is renewed every time a workstation disassociates from the AP.

- Phase 7: All network traffic between the AP and the STA is encrypted.

- Phase 8: When the STA is not authenticated, the AS indicates to the AP the failure of this procedure with EAP-Failure message.

\section{DIGITAL SIGNATURES}

One of the main advantages of asymmetric cryptography is that it offers the possibility of using digital signatures. According to ISO 7498-2, these signatures are used to verify the following properties: the authenticity of the author of an electronic message, but also to ensure its integrity and non repudiation.

The digital signature (Menezes et al., 2001, Chapter 11) proves to the recipient of a message that it has been signed by the author, because it is authentic and difficult to imitate. It is not reusable, and is an integral part of the document.

The Asymmetric Cryptography consists of two asymmetric keys (public / private). The keys are mathematically related. An encryption key and a decryption key are different; one key cannot be calculated from the other.

In general, one of the keys is public and known by everyone. This key will be used by any person to encrypt a message. The original message is extremely difficult to find as it can only be decrypted by the person who possesses the second secret key called the private key.

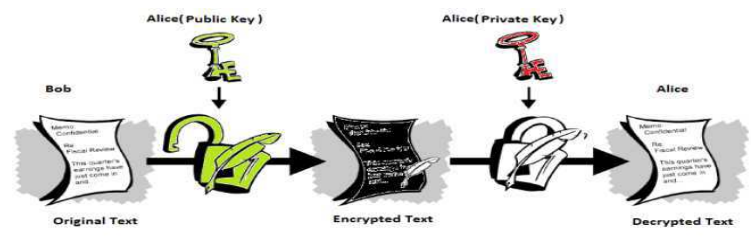

Figure 3: Encryption and Decryption with an Asymmetric Key.

The operation of asymmetric encryption is simple "Figure 3": Bob encrypts the text using the public key of Alice. Alice can decrypt the text with the associated key.

So it is possible to encrypt a message securely with the public key, and only the person with the private key can decrypt it.

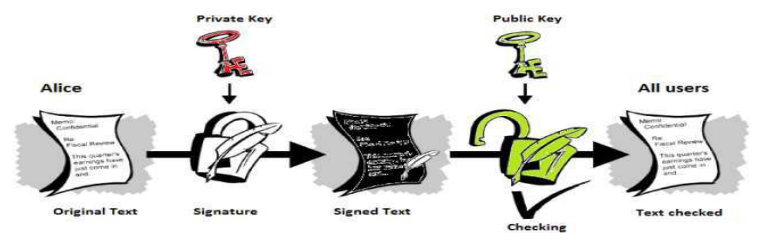

Figure 4: Digital Signature.

The digital signature is the opposite principle of public key encryption "Figure 4". 
Alice encrypts a message with her private key. The result obtained can be decrypted by anyone with the associated public key. This confirms that Alice has signed the content of this message. She cannot repudiate it and can be verified by a third party.

When the document is voluminous, it must be first hashed into just few lines. This process is called a message digest. Then, the message digest must be encrypted using the private key. The result is the digital signature. This digital signature is appended to the document and sent to the recipient.

First, the recipient decrypts the digital signature (using public key) and obtains the message digest. Then, the recipient hashes the received document into a new message digest. If the two message digests are the same, the recipient knows that the signed data has not been changed.

\section{CONTRIBUTIONS}

As mentioned above, in order to obtain an access to a wireless network infrastructure, the STA must be authenticated and must share the MSK secret with a PoA (an AP) "Figure 3". Therefore, the AS has no role and exchanges continue between AP and STA. The AP makes decisions on granting access to the LAN.

Our approach consists to reuse the MSK, shared between the STA and the AP, in each reauthentication to ensure the continuity of the session of the STA.

\subsection{Principle of Virtual ESS (VESS)}

At the beginning of wireless network, the protocol exchanges between STA and AP were much simpler. The packet number, to establish a connection, was mainly of four messages. With secure standards, the number of messages increased considerably.

Our contribution proposes enhancements to IEEE 802.11i standard, which allows a station to switch faster and seamlessly from one AP to another target. The idea is based on the signed token with an Asymmetric Cryptography to establish a trusted infrastructure. This trusted infrastructure can be considered as a wireless network infrastructure of the same ISP "Figure 5" and consists of one or more AS \& AP.

In this large infrastructure of distributed access, we use multiple AS to simplify management and ensure the scalability of the infrastructure.
Each AS handles APs or a Basic Service Set (BSS), which are deployed on the private key. However, the public key is deployed only on APs. We have restricted the deployment of asymmetric keys only to AS and APs.

The private key is used to sign the tokens after each authentication of the STA. The signed token saves the context of a user session and can contain in particular: "Time-Stamps" (to limit the token in time), the message (EAP.Success), and the MSK shared between the STA and the AP. For other uses, this token may also contain: STA attributes, user profile, etc.

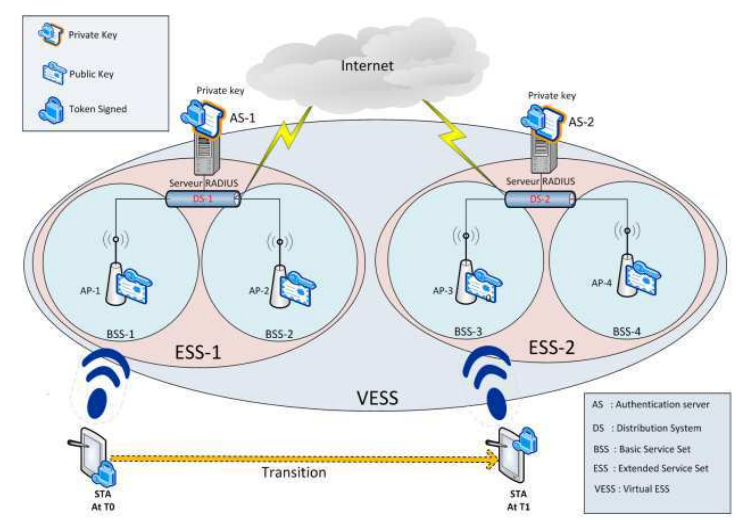

Figure 5: Virtual ESS Infrastructure.

These APs are interconnected by a Distribution System (DS), usually is LAN on which the APs are interconnected. These different BSS interconnected via a DS in the same LAN are called an ESS (Extended Service Set). An ESS is identified by an ESSID.

The V.ESS is an extension of the ESS. It plays the role of an approval authority. In this trusted infrastructure, one of the "AS" signs the token with the private key and the AP decrypts it with the public key.

In other words, when a STA is authenticated by AS-1 in BSS-1 "Figure 5"; this AS-1 signs a token with attributes of the STA, including the MSK negotiated as seen in (Section 4 "Figure 2" Phase 4), and this token is sent via AP-1 to the STA.

When the STA moves to AP-3 in BSS-3, it will present the token. The AP-3 will be able to authenticate the STA, because it possesses the public key of VESS infrastructure. It then decrypts the token to retrieve all attributes, particularly the MSK.

Once the MSK is recovered, it can be reused and derived by AP-3 and STA as seen in (Section 4 "Figure 2" Phase 6). 


\section{Remark 1:}

We notice that the AS-2 in DS-2 is not involved in this process of re-authentication because the reauthentication service has been delegated partially to the APs. This is to avoid the Back-End authentication, and to simplify considerably the number of authentication request between the BackEnd and the STA.

\subsection{Use case: (Re)-authentication Process in VESS}

We illustrate in "Figure 6" the authentication process and, during mobility, the re-authentication process of the STA in VESS infrastructure.
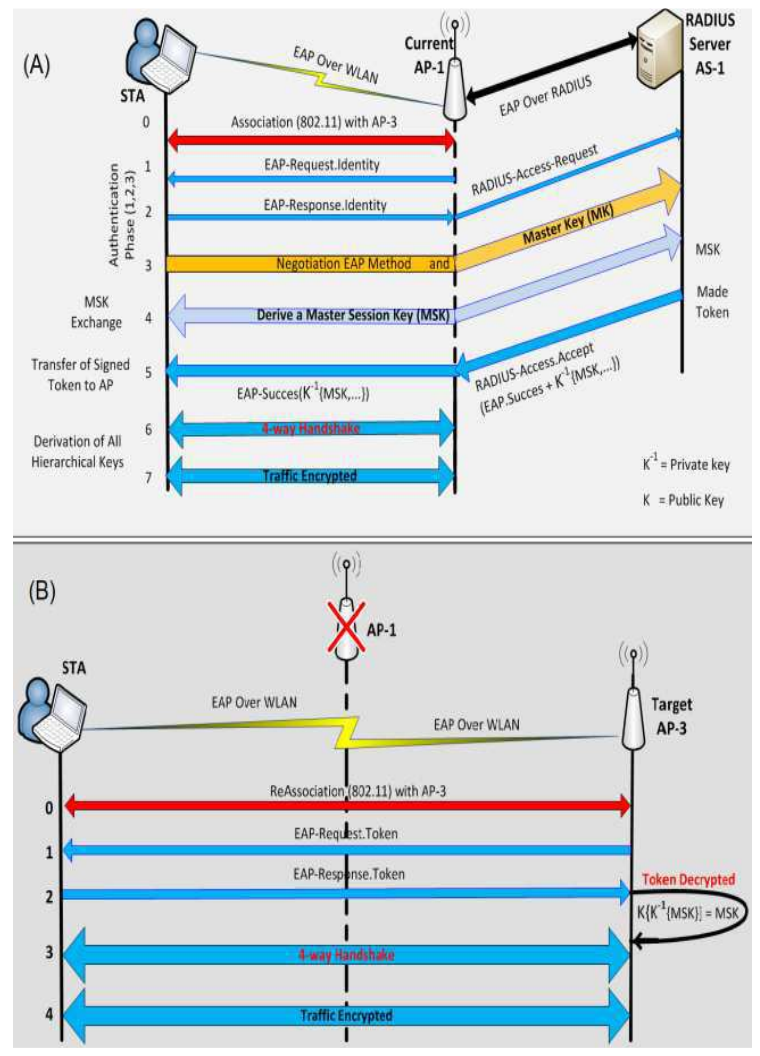

Figure 6: Virtual ESS: Approved Infrastructure.

- Scenario 1: STA authentication with AP-1 "Figure 6 (A)"

Phase 0: during this phase initialization and association, STA and AP-1 negotiate the EAP method with token signed.

Phase 1-2-3-4: authentication of STA and negotiation of the secret key (MSK). In these phases, nothing changes.
Phase 5: AS-1 signs the token containing (attributes, user profile, "Time-stamps", message EAP.Success and MSK) with the private key, and transmits to the AP-1 the token signed in Access.Accept $: \mathrm{K}^{-1}=$ is private key,

Access. Accept (EAP.Success + MSK $+K^{-1}\{$ MSK... $\}$ )

Then the AP-1 forwards the EAP.Success and signed token packet to the STA:

$$
\text { EAP.Success }+K^{-1}\{\text { MSK... }\}
$$

Phase 6: the "4-way handshake" protocol, STA and AP-1 derived from the MSK, a bunch of keys used only in this session with AP-1.

\section{Remark 2:}

In this scenario, "Figure 6 (A)", the exchanges between the STA, AP-1 and AS-1 are almost identical as in "Figure 2". The only difference consists in signing a token after the authentication process of the STA. This signed token ensures continuity of service and the session of the STA.

- Scenario 2: STA re-authentication with AP-3 "Figure 6 (B)"

In this scenario of mobility, the STA transits the AP-1 to AP-3. Phase 0: during this phase reinitialization and re-association, the STA and AP-3 negotiate the EAP method with token signed.

Phase 1-2: STA presents the signed token to the AP-3. The AP-3 decrypts it, using the public key: verifies the authenticity of the token and extract the MSK.

Phase 3: with the "4-way handshake" protocol, AP-3 and STA proceed to the derivation of other keys.

Phase 3: with the "4-way handshake" protocol, AP-3 and STA proceed to the derivation of keys, from the MSK.

The new derivated keyring is different from that obtained with AP-1. This keyring will be used only in this session with AP-3.

\section{Remark 3:}

In this scenario, the STA did not need to initiate a re-authentication process with AS-2, but it was meant only to present a signed token to the AP-3 to be authenticated. With this delegation of the reauthentication process to the AP-3, we avoided the invocation of the AS-2, so we reduced sufficiently the number of requests of EAP authentication; therefore, the Handover latency will be also low. The keys negotiation is done in four passes instantly and seamless. 


\subsection{Justification of our Approach}

In comparison with other existing solutions (IEEE 80211f, 802.11r, etc.), our approach is very easy to implement, can use strong authentication (asymmetric or symmetric cryptography) and does not require many modifications of EAP authentication methods. The implementation of our EAP methods consists in modifying :

- The beacon at the time of the negotiation of the Robust Security Network Information Element (RSN IE) to notify the client $802.1 \mathrm{x}$ that wireless infrastructure supports the negotiation of the tokens signed.

- The requests of (re)-association: the STA 802.11 must notify its choice of security policy by RSN IE included in these requests.

- The Access.Accept method to sign the token and transfer it to the STA and the AP.

\section{CONCLUSION AND PERSPECTIVES}

This text describes the impact of security on the mobility and session continuity. We have identified the limitations of the EAP protocol in combination with the AAA Framework. This standard is used in the access control to different network technologies.

Several stages of the Handover process can be improved. In this paper, we proposed a fast Handover scheme; taking into consideration only delays in re-authentication during a transition in the same VESS.

To do this, we identified our needs in terms of security in relation to mobility. Thus we proposed to implement security components at the APs, to delegate the re-authentication service to APs in the VESS infrastructure.

In our scheme, the AAA Framework is ignored during the process of re-authentication. Therefore, we use a signed token that ensures trust in the VESS and manages mobility and continuity of the single session of STA. This proposition can solve the problem of intercellular delays (see Section II A).

In the next step, our main focus will be to resolve all technical barriers, evaluate the pertinence of our approach to meet the constraints of real-time applications. Finally, we propose improvements to the EAP protocol and we implement the new methods for managing virtual organizations with our signed tokens.

\section{REFERENCES \& STANDARDS}

Bangolae, S., Bell, C. \& Qi, E., 2006, 'Performance study of fast BSS transition using IEEE 802.11r', IWCMC '06 Proceedings of the 2006 international conference on Wireless communications and mobile computing pp. 737-742, 2006.

Da Conceicão, AF., Li, J., Florêncioy, DA., \& Kon, F., 2006, 'Is IEEE 802.11 ready for VoIP?', Department of Computer Science, Institute of Mathematics and Statistics, University of Sào Paulo, Communication and Collaboration Systems, Microsoft Research.

Menezes, AJ., Van Oorschot, PC., \&Vanstone, SA., 2001, 'Handbook of Applied Cryptography', Chapter 11, CRC Press, Fifth Printing August 2001, freely available at $\langle\mathrm{http} / / / \mathrm{cacr}$.uwaterloo.ca/hac/ $\rangle$.

Mishra, A., Shin, M., \& Arbaugh, W., 2003, 'An empirical analysis of the IEEE $802.11 \mathrm{mac}$ layer handoff process' SIGCOMM Comput. Commun. Rev., vol. 33, no. 2, pp. 93-102, 2003.

Velayos, H., \& Karlsson, G., 2004, 'Techniques to reduce the IEEE $802.11 \mathrm{~b}$ handoff time' Tech. Rep., 20-24 June 2004

Zrelli, S. \& Shinoda, Y., 2007, 'Experimental evaluation of EAP performance in roaming scenarios', AINTEC '07 Proceedings of the 3rd Asian conference on Internet Engineering: Sustainable Internet, pp. 86-98, 2007.

AAA Authorization Framework, 2000, IETF RFC 2904, August 2000, <http://tools.ietf.org/html/rfc2904>.

EAP, 2004, 'Extensible Authentication Protocol', IETF RFC 3748, <http://tools.ietf.org/html/rfc3748>, Jun. 2004.

IAPP, 2003, IEEE std 802.11F-2003, 'IEEE Trial-Use Recommended Practice for Multi Vendor Access Point Interoperability Via an Inter Access Point Protocol (IAPP) Across Distribution Systems Supporting IEEE 802.11 Operation'.

IEEE 802.1X, 2001, IEEE Std 802.1X, 'Standards for Local and Metropolitan Area Networks: Port Based Access Control', June 14, 2001.

ISO 7498-2, 1989, 'Information processing systems -Open Systems Interconnection--Basic Reference Model' Part 2: Security Architecture.

RADIUS, 2000, 'Remote Authentication Dial In User Service', IETF Std RADIUS RFC 2865, June 2000, <http://tools.ietf.org/html/rfc2865>.

RFC 2548, 1999, IETF RFC 2548, March 1999, 'Microsoft Vendor-Specific RADIUS Attributes', <https://tools.ietf.org/html/rfc2548>.

RFC 3078, 2001, IETF RFC 3078, March 2001, 'Microsoft Point-To-Point Encryption (MPPE) Protocol', <http://www.ietf.org/rfc/rfc3078.txt>.

WLAN, 1997, IEEE std 802.11, 'WIRELESS LOCAL AREA NETWORKS', The Working Group for WLAN Standards, <http://www.ieee802.org/11/>. 